\title{
Diffraction imaging of organic materials in extreme environments
}

Yujun Xie ${ }^{1}$, Ruopeng Zhang ${ }^{2}$, Colin Ophus ${ }^{3}$, Andrew Minor ${ }^{4}$, Haimei Zheng ${ }^{5}$, Peter Ercius ${ }^{5}$ and Peter Hosemann $^{6}$

${ }^{1}$ Department of Nuclear Engineering, University of California, Berkeley, Berkeley 94720, USA, emeryville, California, United States, ${ }^{2}$ National Center for Electron Microscopy, Molecular Foundry, Lawrence Berkeley National Laboratory, Berkeley 94720, USA, United States, ${ }^{3}$ Lawrence Berkeley National Laboratory, California, United States, ${ }^{4}$ UC Berkeley, Berkeley, California, United States, ${ }^{5}$ Lawrence Berkeley National Laboratory, United States, ${ }^{6}$ University of California, Berkeley, United States

The hidden structures in any organic materials govern their functional properties for wide range applications, including the organic electrolyte in Li-ion battery and kerogen as the source for hydrocarbon production through fracking. However, direct imaging of the structure in organic materials at the level of individual molecules has not been possible using conventional transmission electron microscope (TEM). This challenge is in part due to the highly electron beam sensitivity of organic liquid and their irregular arrangement of low Z contrast elements, i.e., C, H and O. Despite the tremendous progress made in the past few years, imaging of organic materials are limited by: 1) the trade-off between detection range and spatial resolution, 2) the experimental condition set by the cryogen, i.e., liquid nitrogen $(77 \mathrm{~K})$ or Helium $(2 \mathrm{~K})^{1,2}$, and 3) the lack of capability to combine with other in situ techniques, such as mechanical deformation. Therefore, it is of great interest to explore new ideas for improved understanding of the structure-property relationship in organic materials.

Toward this end, we first show that by coupling the Four-Dimensional Scanning Transmission Electron Microscopy (4D-STEM) characterization technique ${ }^{2}$, liquid-phase TEM and variable low temperature system, we are able to image the structural ordering in liquidus organic electrolyte at $-30^{\circ} \mathrm{C}$ that never been achieved in TEM before (Fig 1). For the first time, we are able to distinguish two different liquid phases and one crystallin phase through unsupervised machine learning data processing and realize the enhanced pi-pi stacking ordering of DEC molecules due to the highly concentrated LiPF6.

In the second studies, we will present the first 4D-STEM strain mapping of the fractured kerogen on the Push to Pull (PTP) device through in situ mechanical deformation. We are able to show the essential brittle nature of the mature kerogen (high sp2/sp3 ratio with large number of aromatic rings) during deformation. 4D-STEM strain mapping technique agrees with the theoretical prediction that the strain is in general localize in the aromatic ring region ${ }^{3}$. 


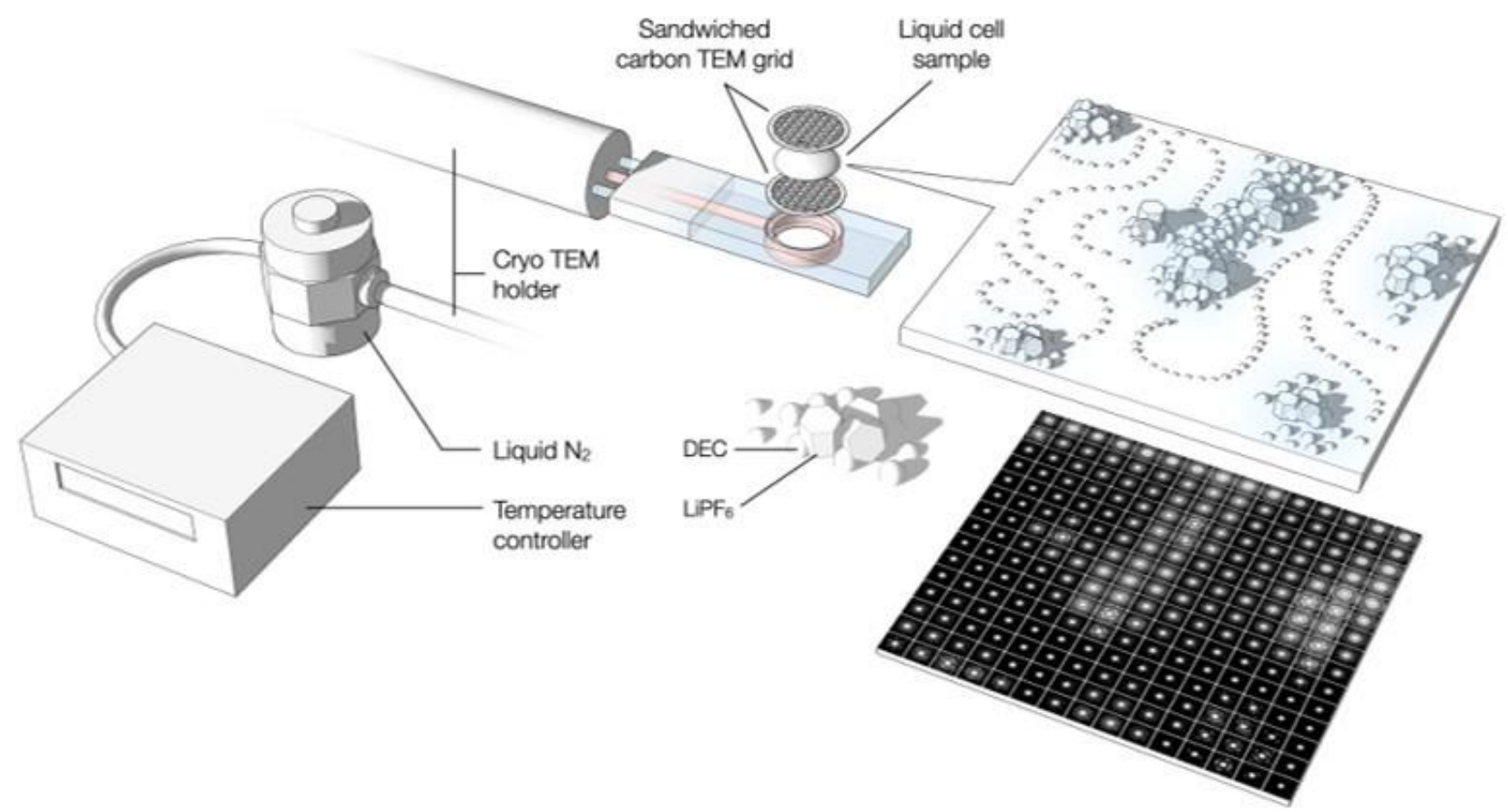

Figure 1. The experimental set up of the 4DSTEM, liquid cell technique and low temperature systems.
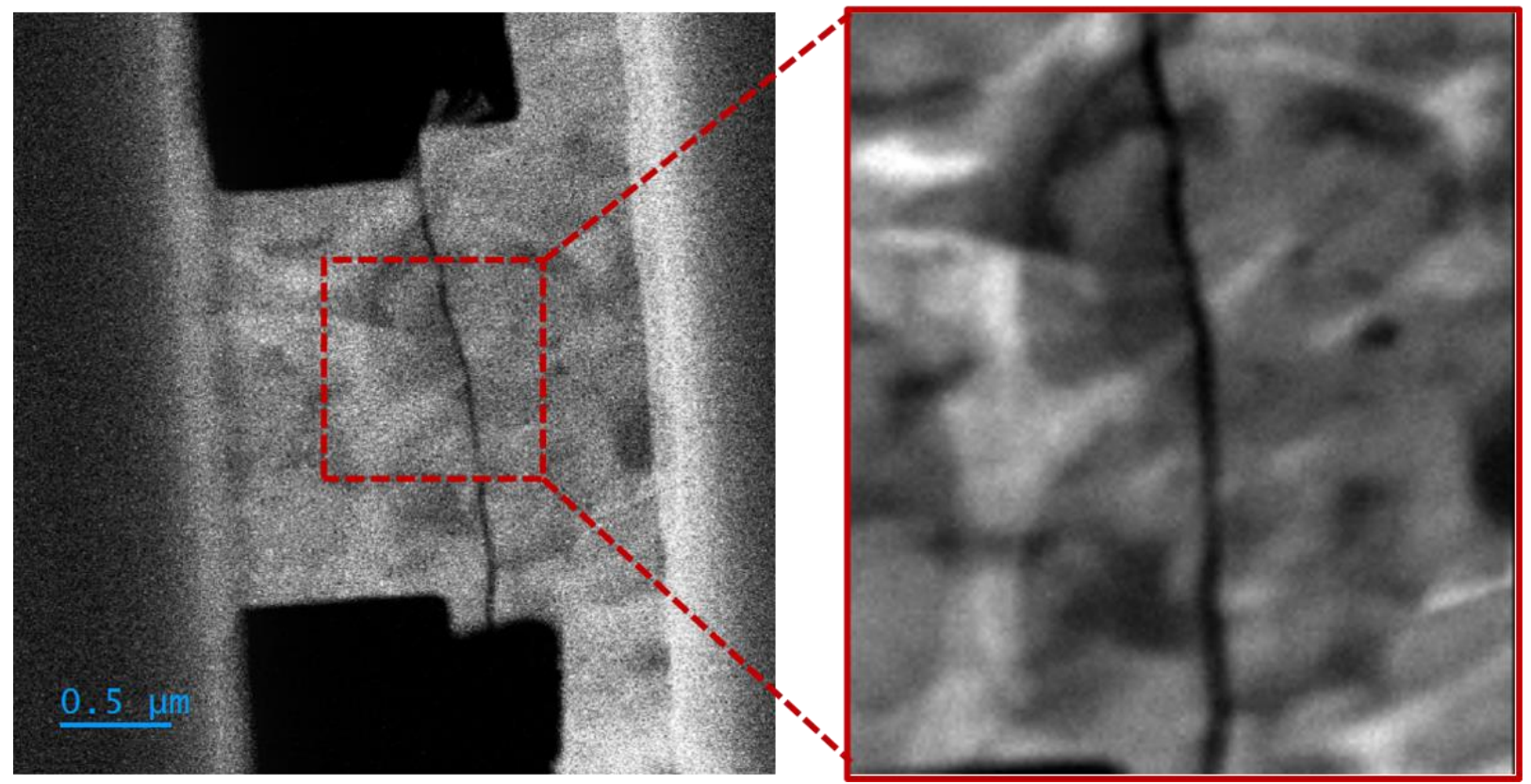

Figure 2. Example of the brittle kerogen on the PTP device.

\section{References}

1) Zachman, Michael J., et al. "Cryo-STEM mapping of solid-liquid interfaces and dendrites in lithiummetal batteries." Nature 560.7718 (2018): 345-349.

2) Panova, Ouliana, et al. "Diffraction imaging of nanocrystalline structures in organic semiconductor molecular thin films." Nature materials 18.8 (2019): 860-865.

3) Bousige, Colin, et al. "Realistic molecular model of kerogen's nanostructure." Nature materials 15.5 (2016): 576-582. 